\title{
Comparison of the Efficacy between First-Line Treatment Regimens for Patients with Hormone Receptor-Positive and HER2-Negative Metastatic Breast Cancer
}

\author{
Kwang Hyun Choi, M.D. ${ }^{*}$, Chan Sub Park, M.D., Ph.D. ${ }^{1 *}$, Sang Hee Kim, M.D. ${ }^{1}$, Na Won Kim, M.D. ${ }^{1}$, \\ Ju Hyeon Lee, M.D. ${ }^{1}$, Ji Yea Kim, Ph.D. ${ }^{2}$, In Chul Park, Ph.D. ${ }^{2}$, Min Ki Seong, M.D., Ph.D.(Cand.) ${ }^{1}$, \\ Hyun-Ah Kim, M.D., Ph.D. ${ }^{1}$, Woo Chul Noh, M.D., Ph.D. ${ }^{3}$ \\ ${ }^{1}$ Department of Surgery, Korea Cancer Center Hospital, ${ }^{2}$ Division of Fusion Radiology Research, Korea Institute of Radiological and Medical Sciences, \\ ${ }^{3}$ Department of surgery, Breast Cancer Center, Konkuk University Medical Center, Seoul, Korea
}

Purpose: Endocrine therapy is the first-line treatment recommended for patients with hormone receptor (HR)-positive, human epidermal growth factor receptor 2 (HER2)-negative metastatic breast cancer without visceral crisis. However, this recommendation has not been followed clinically because of efficacy issues. In this study, the survival of patients with HR-positive/HER2-negative metastatic breast cancer was evaluated based on the following first-line treatment regimens: the combination of palbociclib plus letrozole, conventional endocrine therapy, or chemotherapy. Methods: Medical records were reviewed for this retrospective analysis. Patients with HR-positive/HER2-negative metastatic breast cancer were included. Progression-free survival (PFS) and overall survival (OS) were compared based on first-line treatment regimens. Results: A total of 184 patients were included in the analysis. The first-line treatments were palbociclib plus letrozole in 46 patients (25.0\%), endocrine therapy in 40 patients (21.7\%), and chemotherapy in 98 patients (53.3\%). The PFS of the palbociclib plus letrozole group was significantly longer than that of the endocrine therapy (hazard ratio $=3.43, p<0.001$ ) and chemotherapy (hazard ratio $=2.88$, $p=0.001)$ groups. No significant difference was observed between the endocrine therapy and chemotherapy groups $(p=0.430)$. The OS of the palbociclib plus letrozole group was significantly longer than that of the endocrine therapy (hazard ratio $=5.34, p=0.009$ ) and chemotherapy (hazard ratio 4.23, $p=0.043$ ) groups. No significant difference was observed between the endocrine therapy and chemotherapy groups $(p=0.451)$. Conclusion: The combination regimen of palbociclib and letrozole could be recommended as the first-line treatment of choice in patients with HR-positive/HER2-negative metastatic breast cancer.

Key Words: Breast neoplasms, Cyclin-dependent kinases, Letrozole, Progression-free survival, Survival

\section{INTRODUCTION}

The prognosis of metastatic breast cancer has improved tremendously due to a better understanding of the disease and the development of new drugs stemming from efforts in the field of research. Due to the relatively long survival of patients with metastatic breast cancer, the quality of life should be considered when developing a treatment strategy for these patients. Therefore, physicians should consider im-

Correspondence: Woo Chul Noh, M.D., Ph.D.

Department of surgery, Breast Cancer Center, Konkuk University Medical Center, 120-1 Neungdong-ro, Gwangjin-gu, Seoul 05030, Korea Tel: +82-2-2030-7581, Fax: +82-2-2030-7748, E-mail: 20210415@kuh.ac.kr

*These authors contributed equally to this work.

This study was fully supported by a grant of the Korea Institute of Radiological and Medical Science (KIRAMS), funded by Ministry of Science and ICT (MSIT), Republic of Korea (No. 50547-2021).

Received: Jul 2, 2021 Revised: Aug 25, 2021 Accepted: Sep 10, 2021 proving the quality of life in addition to prolonging survival when selecting the first-line treatment.

The first-line treatment strategy for metastatic breast cancer varies based on the clinical and pathological characteristics, including the hormone receptor (HR) status, human epidermal growth factor receptor 2 (HER2) overexpression status, menopausal status, previous adjuvant treatment, and disease severity. Historically, cytotoxic chemotherapy has been the preferred treatment choice. However, their adverse effects are relatively severe, leading to treatment cessation in up to $10 \%$ of the cases [1]. Therefore, endocrine therapy is recommended as the first-line treatment for patients with HR-positive/HER2-negative metastatic breast cancer without visceral crisis in modern clinical guidelines [2-4]. However, chemotherapy is still one of the preferred choices because of the lack of reliable evidence comparing the efficacy of first-line chemotherapy with that of endocrine therapy. Studies have 
shown that almost half of the patients without visceral crisis received cytotoxic chemotherapy rather than endocrine therapy as an initial treatment regimen $[5,6]$.

Recently, cyclin-dependent kinase 4/6 (CDK 4/6) inhibitors, such as palbociclib, ribociclib, and abemaciclib, have been introduced as the first-line treatment of choice for metastatic disease. The PALOMA-2, a phase III clinical trial, showed that the combination of palbociclib and letrozole as first-line therapy significantly improved the disease-free survival in patients with advanced HR-positive/HER2-negative breast cancer [7-9]. The quality of life also improved with this combination compared with other treatment groups [10].

The approval of the combination of CDK 4/6 inhibitors and endocrine therapy has been gradually increasing in many countries $[6,11]$. In the Republic of Korea, the combination therapy of palbociclib and letrozole in patients with advanced HR-positive/HER2-negative metastatic breast cancer is covered under insurance since 2017 by the $\mathrm{Na}$ tional Health Insurance Service. Therefore, the combination therapy of palbociclib and letrozole, cytotoxic chemotherapy, or conventional endocrine therapy could all be viable treatment options for patients with metastatic HR-positive/HER2-negative disease without financial barriers. However, physicians and patients are still unsure regarding which treatment regimen should be chosen in the clinical setting.

This study aimed to compare the efficacy of the palbociclib and letrozole combination regimen with that of conventional endocrine therapy and chemotherapy in patients with HR-positive/HER2-negative metastatic breast cancer.

\section{METHODS}

\section{Patients}

This retrospective study analyzed the data of patients diagnosed with metastatic breast cancer between January 1998 and December 2019. Distant metastasis was diagnosed by tissue biopsy or radiological methods such as chest and/or abdominal computed tomography, bone scan, whole-body positron emission tomography-computed tomography, or abdominal ultrasonography. The data were collected by chart review, and the included variables were the HR status, HER2 status, age at the time of primary breast cancer diagnosis, age at the time of metastasis, tumor size, lymph node status, first-line systemic treatment, and patient outcomes.
Progression-free survival (PFS) was defined as the time from the start of the first-line treatment to disease progression (confirmed by radiological methods) or death without disease progression. Overall survival (OS) was defined as the time from the start of the first-line treatment to death from any cause. Patients were classified based on the first-line systemic therapy. A visceral crisis was defined as a symptomatic organ dysfunction $[3,12]$. Patients with visceral crisis at the time of diagnosis of metastatic disease were excluded from the analysis (Figure 1). This study was approved by the Institutional Review Board (IRB No. 2019-12-004-003) and conducted in accordance with the Declaration of Helsinki. The need for informed consent was waived because of the retrospective nature of this study.

\section{Statistical analyses}

Continuous variables are presented as means \pm standard deviations and medians (interquartile ranges), whereas categorical variables are presented as frequencies and percentages. For comparison of the clinicopathological features between the patient groups, analysis of covariance and likelihood ratios were used depending on the variables. Survival rates were calculated using the Kaplan-Meier method, and differences were assessed using the log-rank test. Statistical significance was set at $p<0.050$.

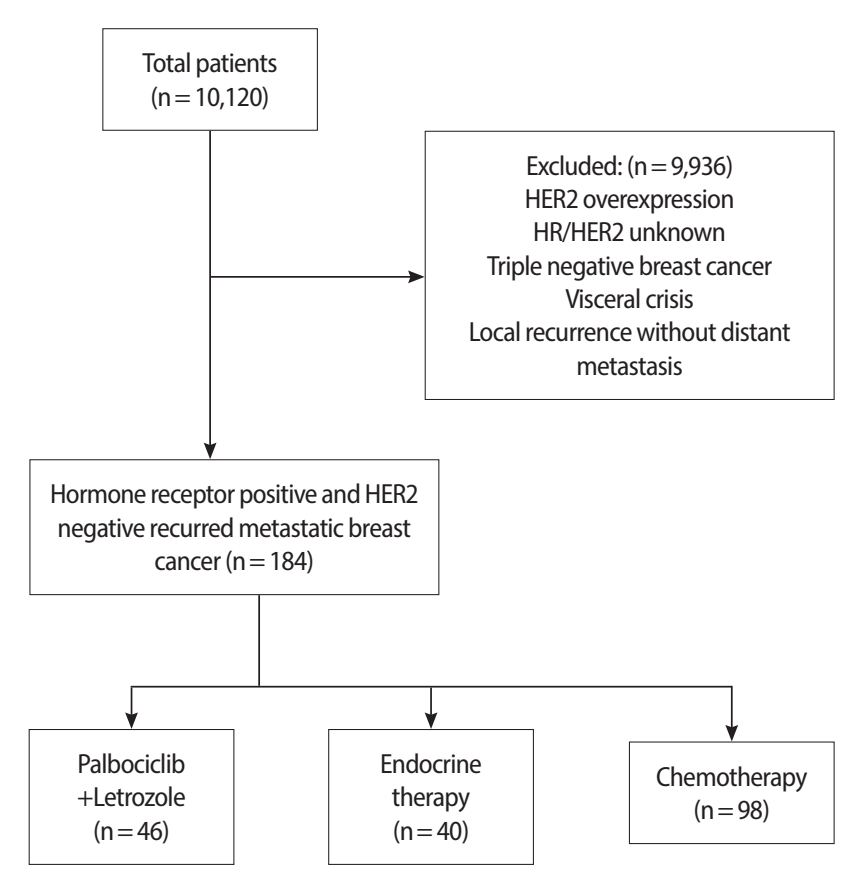

Figure 1. Inclusion and exclusion criteria of the study patients. 
Table 1. Characteristics of study patients

\begin{tabular}{|c|c|c|c|c|}
\hline Characteristic & $\begin{array}{l}\text { Palbociclib + Letrozole } \\
\qquad \begin{array}{c}(n=46) \\
\text { No. }(\%)\end{array}\end{array}$ & $\begin{array}{l}\text { Endocrine Therapy } \\
\qquad \begin{array}{l}(n=40) \\
\text { No. }(\%)\end{array}\end{array}$ & $\begin{array}{l}\text { Chemotherapy } \\
\qquad \begin{array}{c}(n=98) \\
\text { No. }(\%)\end{array}\end{array}$ & $p$-value \\
\hline Age at diagnosis $(y r)^{*}$ & $49.3 \pm 11.3$ & $53.8 \pm 14.2$ & $49.3 \pm 9.6$ & 0.083 \\
\hline Age at metastasis (yr)* & $55.4 \pm 10.6$ & $55.6 \pm 13.4$ & $50.6 \pm 9.2$ & 0.008 \\
\hline \multicolumn{5}{|l|}{ Metastatic status } \\
\hline De novo stage IV & $14(30.4)$ & $25(62.5)$ & $77(78.6)$ & $<0.001$ \\
\hline Metastasis during follow-up & $32(69.6)$ & $15(37.5)$ & $21(21.4)$ & \\
\hline \multicolumn{5}{|l|}{ Metastatic site } \\
\hline Visceral & $19(41.3)$ & $18(45.0)$ & $51(52.0)$ & 0.194 \\
\hline Non-visceral & $14(30.4)$ & $7(17.5)$ & $13(13.3)$ & \\
\hline Bone-only & $13(28.3)$ & $15(37.5)$ & $34(34.7)$ & \\
\hline Follow up periods (mo) ${ }^{*}$ & $25.0 \pm 1.3$ & $40.0 \pm 4.0$ & $44.7 \pm 3.8$ & 0.002 \\
\hline
\end{tabular}

*Mean \pm SD.

Table 2. Regimen details of chemotherapy $(n=98)$

\begin{tabular}{lcc}
\hline \multirow{2}{*}{ Regimen } & \multicolumn{2}{c}{ Frequency } \\
\cline { 2 - 3 } & No. & $(\%)$ \\
\hline Anthracycline+Cyclophosphamide & 6 & $(6.1)$ \\
Anthracycline+Cyclophosphamide followed by Taxane & 7 & $(7.1)$ \\
Anthracycline+ Taxane & 70 & $(71.4)$ \\
Capecitabine & 4 & $(4.1)$ \\
Oral 5-Fluorouracil & 1 & $(1.0)$ \\
Docetaxel + Cisplatin & 2 & $(2.2)$ \\
5-Fluorouracil + Anthracycline+Cyclophosphamide & 7 & $(7.1)$ \\
Gemcitabine + Cisplatin & 1 & $(1.0)$ \\
\hline
\end{tabular}

\section{RESULTS}

\section{Patient characteristics}

A total of 184 patients were included in the analysis. A total of 46 patients received a combination of palbociclib and letrozole, $40 \mathrm{pa}-$ tients received endocrine therapy, and 98 patients received chemotherapy, respectively. The mean age at the initial diagnosis of primary breast cancer was $50.4 \pm 11.2$ years, and the mean age of metastasis was $53.2 \pm 11.0$ years. In the conventional endocrine therapy group, these ages were $53.8 \pm 14.2$ years and $55.6 \pm 13.4$ years, respectively. The mean age of metastasis in the endocrine therapy group was significantly higher than that in the other groups. Visceral metastasis was observed in $41.3 \%$ of the patients in the palbociclib plus letrozole group, $45.0 \%$ of those in the conventional endocrine therapy group, and $52.0 \%$ of those in the chemotherapy group. The mean follow-up period was $25.0 \pm 1.3$ months in the palbociclib plus letrozole group, $40.0 \pm 4.0$ months in the endocrine therapy group, and $44.7 \pm 3.8$ months in the chemotherapy group (Table 1).
Table 3. Regimen details of endocrine therapy $(n=40)$

\begin{tabular}{lrc}
\hline \multirow{2}{*}{ Regimen } & \multicolumn{2}{c}{ Frequency } \\
\cline { 2 - 3 } & No. & $(\%)$ \\
\hline Aromatase inhibitor & 25 & $(62.5)$ \\
Aromatase inhibitor + Everolimus & 6 & $(15.0)$ \\
Tamoxifen & 9 & $(22.5)$ \\
\hline
\end{tabular}

\section{First-line systemic therapy}

In terms of CDK 4/6 inhibitors, the palbociclib plus letrozole regimen alone was used. In the chemotherapy group, 70 patients $(71.4 \%)$ were treated with anthracycline plus taxane, 6 patients (6.1\%) received anthracycline plus cyclophosphamide, and 7 patients (7.1\%) received anthracycline plus cyclophosphamide followed by taxane (Table 2). Half of the patients who had been treated with chemotherapy $(n=49)$ also received maintenance therapy with aromatase inhibitors $(\mathrm{n}=30)$, tamoxifen $(n=16)$, or capecitabine/oral 5-fluorouracil $(n=3)$. For those who had received endocrine therapy, aromatase inhibitors were administered to 25 patients (62.5\%), aromatase inhibitors plus everolimus were administered to 6 patients (15.0\%), and tamoxifen was administered to 9 patients (22.5\%) (Table 3).

\section{Survival analysis by the treatment type}

The median follow-up period was 30.4 months (range, 1-200 months). The PFS of the palbociclib plus letrozole group was longer than that of the conventional endocrine therapy (hazard ratio $=3.43$, $p<0.001$ ) and chemotherapy (hazard ratio $=2.88, p=0.001$ ) groups (Figure 2). No significant difference in the PFS was observed between the conventional endocrine therapy and chemotherapy groups 


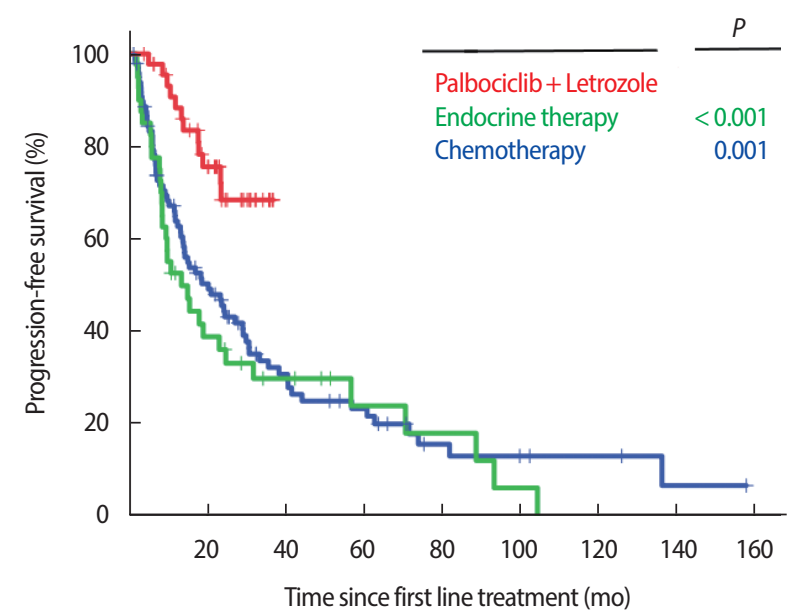

Figure 2. Progression-free survival by the treatment regimen.

$(p=0.430)$.

The OS of the palbociclib plus letrozole group was significantly longer than that of the endocrine therapy (hazard ratio $=5.34, p=0.009$ ) and chemotherapy (hazard ratio $=4.23, p=0.043$ ) groups. No significant difference in the OS was observed between the conventional endocrine therapy and chemotherapy groups $(p=0.451)$ (Figure 3 ).

\section{DISCUSSION}

In this study, patients treated with the palbociclib plus letrozole regimen had a significantly longer PFS and OS than those treated with conventional endocrine therapy or chemotherapy as a first-line systemic treatment for HR-positive/HER2-negative metastatic breast cancer. There was no significant difference in the PFS and OS between the conventional endocrine therapy and chemotherapy groups. To the best of our knowledge, this is the first report of real-world data comparing the survival among those who received palbociclib plus letrozole, conventional endocrine therapy, and chemotherapy as first-line systemic therapy.

Palbociclib is the first CDK 4/6 inhibitor to be used in the clinical setting, and the palbociclib plus letrozole regimen is covered by the National Health Insurance Service in the Republic of Korea since 2017. For this reason, the rate of de novo stage IV disease is higher in the chemotherapy group. Although other CDK 4/6 inhibitors have been added recently, palbociclib alone was available without financial barriers between 1998 and 2019. As the palbociclib plus letrozole regi-

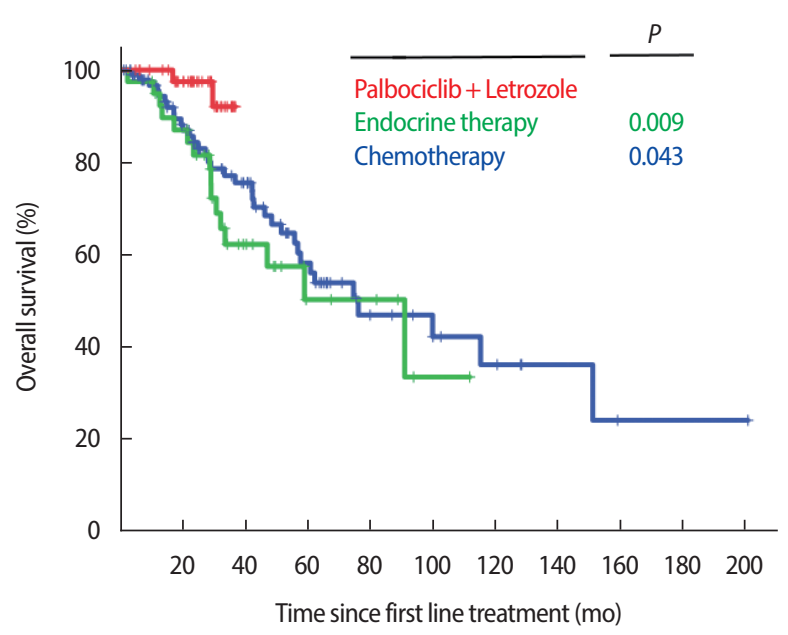

Figure 3. Overall survival by the treatment regimen.

men is a relatively new treatment strategy, the median follow-up period for this group was shorter than that of the others. This group also showed a significant improvement in the PFS and OS compared with the other groups. These results are consistent with those of the PALOMA-2 clinical trial and other real-world data. In the PALOMA-2 trial, the median PFS was prolonged by 10.3 months in the palbociclib plus letrozole group compared with the placebo plus letrozole group (hazard ratio $=0.58, p<0.001$ ) [13]. The survival benefit of palbociclib plus letrozole was also observed in a study with a longer follow-up [7].

Concerns about the reproducibility of clinical trials conducted in well-controlled environments in real-world practice are prevalent among clinicians and patients. Recently, a few researchers have reported real-world clinical experiences with palbociclib. DeMichele et al. [14] reported a retrospective analysis of 1,430 patients and showed better outcomes in the palbociclib plus letrozole group with a PFS of 20.2 months compared with 11.9 months in the letrozole-only group. The median PFS of our study was $30.0 \pm 1.7$ months, which was consistent with previous prospective randomized clinical trials [9]. When combined with our study, the results of the PALOMA-2 trial are reproducible in real-world clinical practice.

Moreover, our study reported no difference in the PFS and OS between conventional endocrine therapy and chemotherapy. Due to financial obstacles, as well as the side effects of neutropenia and thrombocytopenia, CDK 4/6 inhibitors are not the first-line treatment of choice for certain patients. Considering the more severe adverse events associated with chemotherapy, conventional endocrine thera- 
py should be an alternative in these patients.

The limitation of this study is the relatively short follow-up period of the palbociclib and letrozole group compared with that of the other groups because of the recent reimbursement policy for palbociclib since 2017. Although the follow-up duration was relatively shorter than that of the other groups, the significant survival benefit of the palbociclib plus letrozole regimen was clearly demonstrated in this analysis. The study population included patients with metastatic HR-positive/HER2-negative disease without visceral crisis, and breast cancer with these characteristics is known to have a relatively better prognosis compared with other subtypes. Our previous research showed that HR-positive and HER2-negative breast cancer patients showed a long-term stable disease status. Therefore, if the follow-up period is longer, patients belonging to the palbociclib plus letrozole group could have a long-term stable disease status [15].

In conclusion, the palbociclib plus letrozole regimen could be recommended as the first-line treatment of choice in patients with HR-positive/HER2-negative metastatic breast cancer without visceral crisis based on its efficacy in real-world clinical practice. In patients who cannot receive palbociclib or other CDK 4/6 inhibitors for any reason, conventional endocrine therapy could be a more reasonable alternative to chemotherapy in terms of the quality of life.

\section{CONFLICT OF INTEREST}

The authors declare that they have no competing interests.

\section{REFERENCES}

1. Jacquet E, Lardy-Cleaud A, Pistilli B, Franck S, Cottu P, Delaloge S, et al. Endocrine therapy or chemotherapy as first-line therapy in hormone receptor-positive HER2-negative metastatic breast cancer patients. Eur J Cancer 2018;95:93-101.

2. Rugo HS, Rumble RB, Macrae E, Barton DL, Connolly HK, Dickler $\mathrm{MN}$, et al. Endocrine therapy for hormone receptor-positive metastatic breast cancer: American Society of Clinical Oncology Guideline. J Clin Oncol 2016;34:3069-103.

3. Paluch-Shimon S, Cardoso F, Sessa C, Balmana J, Cardoso MJ, Gilbert F, et al. Prevention and screening in BRCA mutation carriers and other breast/ovarian hereditary cancer syndromes: ESMO
Clinical Practice Guidelines for cancer prevention and screening. Ann Oncol 2016;27:v103-10.

4. Gradishar WJ, Anderson BO, Abraham J, Aft R, Agnese D, Allison KH, et al. Breast cancer, version 3.2020, NCCN Clinical Practice Guidelines in Oncology. J Natl Compr Canc Netw 2020;18:452-78.

5. Swallow E, Zhang J, Thomason D, Tan RD, Kageleiry A, Signorovitch J. Real-world patterns of endocrine therapy for metastatic hormone-receptor-positive $(\mathrm{HR}+)$ /human epidermal growth factor receptor-2-negative (HER2-) breast cancer patients in the United States: 2002-2012. Curr Med Res Opin 2014;30:1537-45.

6. Fietz T, Tesch H, Rauh J, Boller E, Kruggel L, Janicke M, et al. Palliative systemic therapy and overall survival of 1,395 patients with advanced breast cancer - results from the prospective German TMK cohort study. Breast 2017;34:122-30.

7. Rugo HS, Finn RS, Dieras V, Ettl J, Lipatov O, Joy AA, et al. Palbociclib plus letrozole as first-line therapy in estrogen receptor-positive/ human epidermal growth factor receptor 2-negative advanced breast cancer with extended follow-up. Breast Cancer Res Treat 2019;174:719-29.

8. Rossi V, Berchialla P, Giannarelli D, Nistico C, Ferretti G, Gasparro S, et al. Should all patients with HR-positive HER2-negative metastatic breast cancer receive CDK 4/6 inhibitor as first-line based therapy? A network meta-analysis of data from the PALOMA 2, MONALEESA 2, MONALEESA 7, MONARCH 3, FALCON, SWOG and FACT trials. Cancers 2019;11:1661.

9. Im SA, Mukai H, Park IH, Masuda N, Shimizu C, Kim SB, et al. Palbociclib plus letrozole as first-line therapy in postmenopausal Asian women with metastatic breast cancer: results from the phase III, randomized PALOMA-2 study. J Glob Oncol 2019;5:1-19.

10. Finn RS, Crown JP, Lang I, Boer K, Bondarenko IM, Kulyk SO, et al. The cyclin-dependent kinase 4/6 inhibitor palbociclib in combination with letrozole versus letrozole alone as first-line treatment of oestrogen receptor-positive, HER2-negative, advanced breast cancer (PALOMA-1/TRIO-18): a randomised phase 2 study. Lancet Oncol 2015;16:25-35.

11. Husinka L, Koerner PH, Miller RT, Trombatt W. Review of cyclin-dependent kinase $4 / 6$ inhibitors in the treatment of advanced or metastatic breast cancer. J Drug Assess 2021;10:27-34.

12. Cardoso F, Costa A, Senkus E, Aapro M, Andre F, Barrios CH, et al. 3rd ESO-ESMO international consensus guidelines for advanced 
breast cancer (ABC 3). Breast 2017;31:244-59.

13. Finn RS, Martin M, Rugo HS, Jones S, Im SA, Gelmon K, et al. Palbociclib and letrozole in advanced breast ancer. N Engl J Med 2016;375:1925-36.

14. DeMichele A, Cristofanilli M, Brufsky A, Liu X, Mardekian J, McRoy L, et al. Comparative effectiveness of first-line palbociclib plus letrozole versus letrozole alone for HR+/HER2- metastatic breast cancer in US real-world clinical practice. Breast Cancer Res 2021;23:37.

15. Noh YH, Kim YG, Kim JH, Choi HS, Lee SJ, Kim JS, et al. Factors associated with metastatic breast cancer in patients who show longterm stable disease status. J Breast Dis 2017;5:1-7. 